\title{
Mobility in Wireless Networks
}

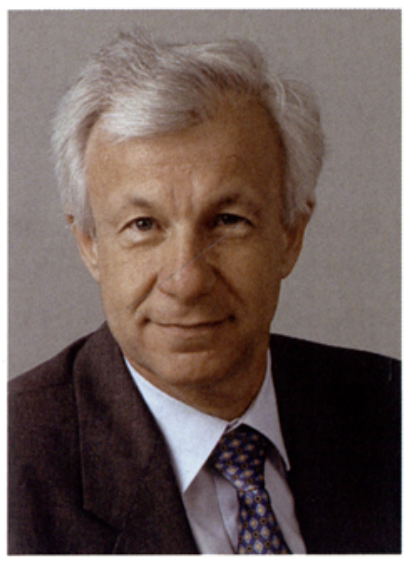

Dr. Helmut Malleck

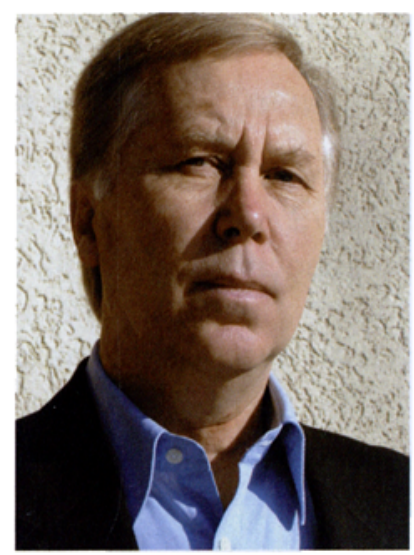

Prof. Dr. Reinhold Weiss

\section{Liebe Leser!}

Mobilkommunikation ist heute mehr denn je Motor der gesamten Telekommunikationsbranche, indem immer weitreichendere Mobilitätsbedürfnisse aus dem Privat- und Wirtschaftsleben, aus Industrie und Technik erfüllbar werden. Die ökonomische Entwicklung insgesamt wird damit positiv beeinflusst. Wesentlichen Anteil haben dabei die zellularen Mobilfunknetze. Nicht minder bedeutend sind Mobility in Wireless Networks und Wireless Networking, welche sich im letzten Jahrzehnt zu eigenständigen Disziplinen entwickelt haben.

Vor einem halben Jahr hat Österreich mit der Umsetzung des visionären Konzepts der dritten Mobilfunkgeneration UMTS eine namhafte Stelle innerhalb der EU erreicht, und noch in diesem Jahr sollen mit der neuen Handy-Generation von mehreren Anbietern UMTS-Dienste in den Ballungsräumen für Experten bereitgestellt werden. Deutschland, Spanien, Großbritannien und Frankreich unternehmen ebenfalls große Anstrengungen, die UMTS-Versorgung voranzubringen.

Treibende Kräfte für die Erfolge der Mobilkommunikation sind auf der einen Seite die heutige Mikrotechnologie und die in die Zukunft weisende Nanotechnologie mit der Entwicklung von Signalprozessoren hoher Leistungsfähigkeit und geringem Energiebedarf. Damit wurde die Voraussetzung für mobile, tragbare und vernetzbare Kleinrechner geschaffen. Andererseits führten neue Konzepte für den Informationstransport, wie Wireless Local Area Networks WLAN und Wireless Personal Area Networks WPAN, Weiterentwicklungen der digitalen Signalver-

arbeitung und nicht zuletzt innovative Hard- und Softwarearchitekturen für verteilte Systeme in Verbindung mit IP-basierten Softwaretechniken zu völlig neuen Anwendungen. Technisch und wirtschaftlich überzeugende Beispiele werden hierzu in den Bereichen Mobile and Nomadic Computing, Ubiquitous Computing and Pervasive Computing sowie Wearable Computing angesiedelt sein.

Als Mobility-Anwendungen stehen M-Commerce, mit mobilem Büro und mobile banking, ebenso wie mobile LANs mit unterschiedlichsten Funktionalitäten heute im Einsatz und erfreuen sich steigender Beliebtheit. Bei den zellularen Mobilfunknetzen sind die Mobility-Dienste Short Message Service SMS und Multi Media Messaging MMS besonders hervorzuheben. Zu UMTS sind Produkte und Services mit Audio Video Streaming in Erprobung.

Es ist uns nun eine besondere Freude, Ihnen mit den Beiträgen des vorliegenden Heftes sowohl Erreichtes als auch die mannigfaltigen Herausforderungen zum Thema Mobility in Wireless Networks in Zusammenhang mit zellularen Mobilfunknetzen präsentieren zu dürfen.

Das multinationale IST-Projekt FLOWS gibt einen richtungweisenden Einstieg, gefolgt von einer Originalarbeit zu LOMOT, einem auf Radio Frequency Tag-Technologie und Bluetooth basierenden System zur Inspektion von komplexen Produktionsanlagen. Mit LoL@ wird ein prototypischer UMTS-Dienst, der einen Touristenführer für die Innenstadt von Wien implementiert, dargestellt. OMAP, eine Kombination aus RISC- und Signalprozessorarchitektur, die sich besonders für mobile Endgeräte eignet, rundet den Originalarbeitsteil des Schwerpunktsheftes ab.

Die Rubrik Praxis\&Wissen berichtet über außerordentliche Leistungen zu Mobility in Wireless Networks: VECAS als mobiles, tragbares Multisensorsystem zur Überwachung von Motorsportveranstaltungen; DiV als Data in Voice-System im Bereich der Flugsicherung; ADONIS als digitales Funksystem für die Blaulichtorganisationen Österreichs und Plattform für eine Vielzahl von Datenapplikationen sowie das innovative, in Wien entwickelte HiPath-Feature für den Handy-Zugriff auf firmeninterne Telefonbücher und zur Fernsteuerung von Anrufumleitungen.

Wir wünschen Ihnen viel Vergnügen bei der Lektüre.
Helmut Malleck OVE<smiles>CC=C=C=CCCC</smiles>

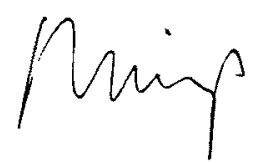

Reinhold Weiss OVE 